\title{
Fallbericht zu schweren multiplen Bronchusstenosen bei einem Patienten, der «das Unüberlebbare überlebtes
}

\author{
Thomas Crowhurst ${ }^{\mathrm{a}, \mathrm{b}}$ Joshua Lightfoot ${ }^{c}$ Aeneas Yeo $^{\mathrm{a}, \mathrm{b}}$ Benjamin Reddic, $\mathrm{d}$ \\ Phan Nguyen $^{a, b}$ Helen Whitford $^{e}{ }^{f} \quad$ Chien-Li Holmes-Liew ${ }^{a, b}$ \\ ${ }^{a}$ Discipline of Medicine, University of Adelaide, Adelaide, SA, Australien; \\ ${ }^{b}$ Department of Thoracic Medicine, Central Adelaide Local Health Network, Royal Adelaide Hospital, Adelaide, SA, Australien; \\ 'Intensive Care Unit, Central Adelaide Local Health Network, Royal Adelaide Hospital, Adelaide, SA, Australien; \\ ${ }^{\mathrm{d}}$ Discipline of Acute Care Medicine, University of Adelaide, Adelaide, SA, Australien; \\ eDepartment of Respiratory Medicine, The Alfred Hospital, Melbourne, VIC, Australien; \\ ${ }^{f}$ Department of Medicine, Monash University, Wellington Road, Clayton, VIC, Australien
}

\section{Schlüsselwörter}

Inhalationsverletzung · Extrakorporale Membranoxygenierung .

Multiple netzartige Bronchusstenosen · Interventionelle

Pneumologie $\cdot$ Lungentransplantation

\section{Zusammenfassung}

Hintergrund: In der Literatur werden nur wenige Fälle von multiplen Bronchusstenosen beschrieben, und keiner von vergleichbarer Schwere wie der unsere. Dieser Fall ist von Relevanz aufgrund seiner Seltenheit, der zu gewinnenden pathophysiologischen Erkenntnisse, der erfolgreich angewandten interventionell-pneumologischen Behandlungsstrategien, und seiner möglichen Beispielrolle für eine seltene Indikation für eine Hochrisiko-Lungentransplantation.

Vorstellung des Falls: Bei einem 47-jährigen Mann traten multiple rezidivierende netzartige Bronchusstenosen auf, 5 Wochen nachdem er eine Episode schwerer Tracheobronchitis durchgemacht hatte, die vermutlich durch eine chemische Inhalationsverletzung verursacht worden war und zunächst zum vollständigen bilateralen Lungenkollaps geführt und eine veno-venöse extrakorporale Membranoxygenierung erforderlich gemacht hatte. Die Stenosen verschlossen die Bronchien an vielen Stellen vollständig und verursachten schwere Typ-II-Ateminsuffizienz, sodass maschinelle Beatmung und eine bronchoskopische Punktion und Dilatation sowie letztlich eine bilaterale Lungentransplantation vorgenommen wurden.

Schlussfolgerung: Dieser sehr seltene Fall unterstreicht die Folgemorbidität, die eine verheerende Tracheobronchitis nach sich ziehen kann, wenngleich sie heute im Zeitalter der extrakorporalen Membranoxygenierung kurzfristig überlebt werden kann.

(c) 2019 Die Autoren)

\section{Hintergrund}

Bronchusstenosen entstehen meist infolge von Tumoren, Infektionen (z.B. Tuberkulose) oder Autoimmunerkrankungen, allerdings in der Regel an einer oder einigen wenigen Stellen $[1,2,3]$. Nur sehr selten ist bisher über multiple Stenosen der Atemwege infolge einer Inhalationsverletzung berichtet worden [4]. In diesem Bericht wird ein seltener Fall von multiplen, rezidivierenden Bronchusstenosen vorgestellt, welche zu schwerer Ateminsuffizienz führten und nur durch aggressive interventionelle pulmonale Behandlungsstrategien und letztlich eine bilaterale Lungentransplantation zu beherrschen waren. information@karger.com

() 2020 S. Karger GmbH, Freiburg

www.karger.com/kkp

Karger ${ }^{\prime \prime}=$
Dr. Thomas Crowhurst

Department of Thoracic Medicine

Central Adelaide Local Health Network, Royal Adelaide Hospital

1 Port Road, Adelaide, SA 5000, Australien

thomas.crowhurst@gmail.com 


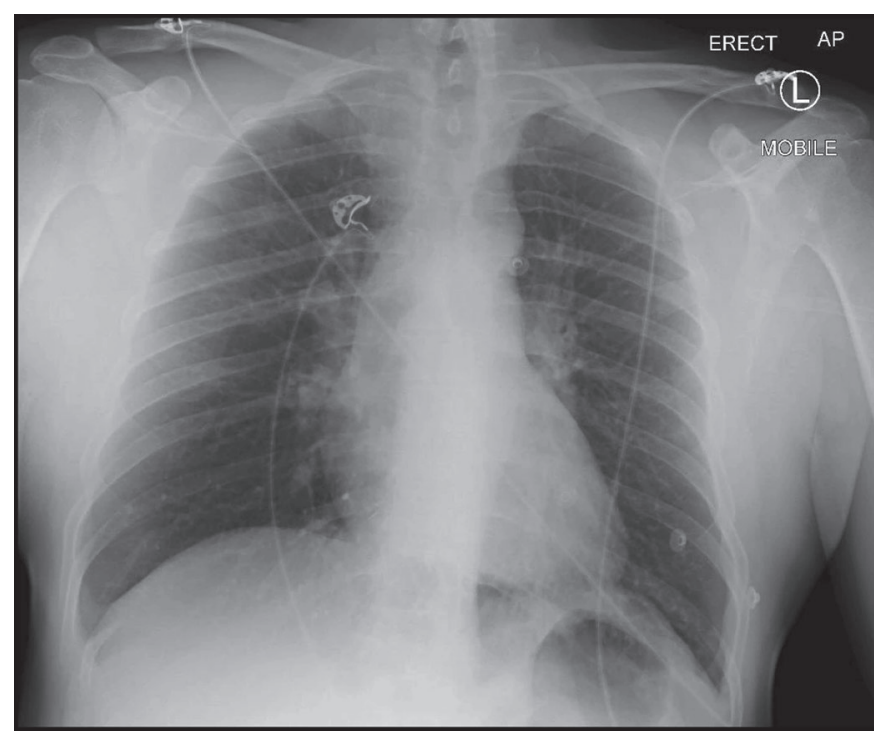

Abb. 1. Effektiv klare konventionelle Thoraxröntgenaufnahme anteroposterior im Stehen bei der Erstvorstellung.

\section{Vorstellung des Falls}

Ein 47-jähriger männlicher Elektriker aus dem ländlichen Australien mit bis dahin gutem Gesundheitszustand stellte sich mit seit 5 Tagen zunehmender Symptomatik von Dyspnoe, produktivem Husten und geringfügiger Hämoptyse vor, die auf orale Antibiotika und Kortikosteroide nicht ansprach. In der Anamnese lagen Depressionen (Desvenlafaxin) und aktiver Raucherstatus (25 Packungsjahre) vor. Das initiale Thoraxröntgen war unauffällig. Im Laufe des folgenden Tages verschlechterte sich sein $\mathrm{Zu}-$ stand rapide zu einer schweren Typ-I-Ateminsuffizienz, welche eine Intubation und maschinelle Beatmung erforderlich machte. Eine ausgeprägte Strömungsbehinderung in den Luftwegen bei der In- und Exspiration verhinderte einen ausreichenden Gasaustausch, daher wurde notfallmäßig eine venovenöse extrakorporale Membranoxygenierung (extracorporeal membrane oxygenation, ECMO) eingeleitet. Im Laufe des nächsten Tages trat ein vollständiger bilateraler Lungenkollaps ein (Abb. 1 und Abb. 2).

Dass im initialen Thoraxröntgen keine entsprechende Luftraumverschattung vorlag, ließ ein akutes Atemnotsyndrom ausschließen und deutete darauf hin, dass die schwere Strömungsbehinderung auf eine Atemwegsobstruktion zurückzuführen sein könnte. Die Bronchoskopie bestätigte eine schwere diffuse Entzündung der Atemwege, die durch weiträumige eitrige Exsudation, Ulzera und Noduli gekennzeichnet war (Abb. 3). Endobronchiale Biopsien ergaben einen akuten nekrotisch-inflammatorischen Prozess. Umfangreiche mikrobiologische Untersuchungen verliefen negativ, lediglich Rhinoviren konnten mittels Polymerase-Kettenreaktion nachgewiesen werden. Auch ein Vaskulitis-Screening einschließlich des antineutrophilen zytoplasmatischen Antikörpers (ANCA) war negativ. Die Verdachtsdiagnose lautete auf Inhalationsverletzung.

Die Behandlung umfasste eine antimikrobielle Breitbandtherapie (mit Meropenem, Vancomycin, Doxycyclin und Voriconazol), wie-

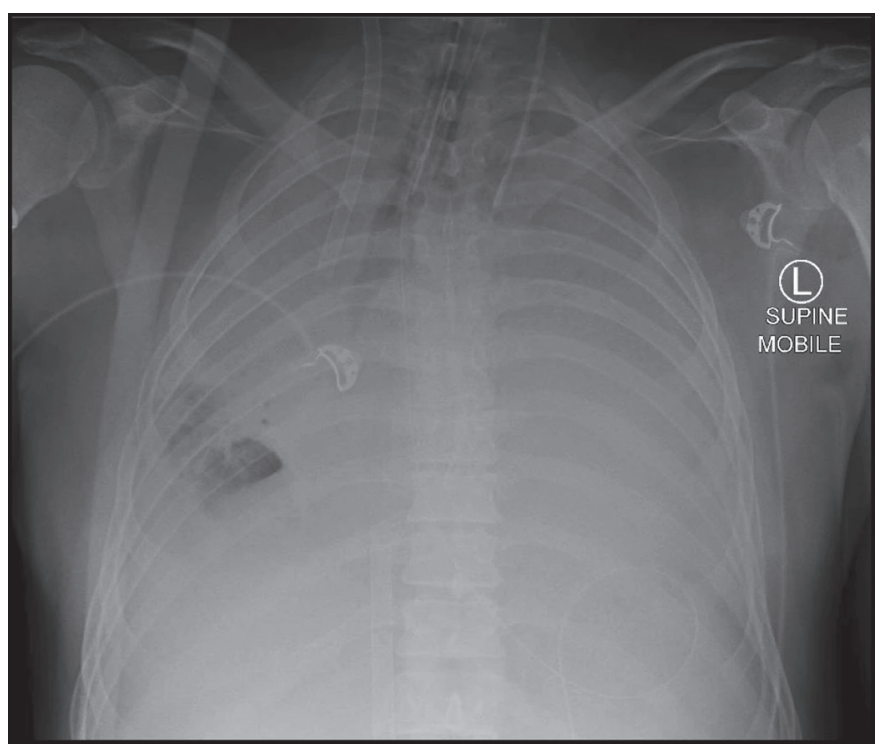

Abb. 2. Konventionelle Intervall-Thoraxröntgenaufnahme anteroposterior in Rückenlage mit vollständigem bilateralem Lungenkollaps 3 Tage nach der Erstvorstellung und einen Tag nach Einleitung einer extrakorporale Membranoxygenierung.

derholte bronchoskopische Toilette und supportive Maßnahmen. Die Atemwegsentzündung besserte sich und nach 13 Tagen, als nur noch eine leichte Strömungsbehinderung in der Exspiration vorlag, wurde auf maschinelle Beatmung umgestellt. Zwei Tage nach Beendigung der ECMO wurde eine Tracheostomiekanüle gelegt, und das Weaning von der Beatmung wurde nach 7 Tagen abgeschlossen. An Tag 24 nach der Aufnahme wurde der Patient auf Station verlegt. Die einzige Komplikation war eine Critical-Illness-Myopathie. Drei Tage später wurde der Patient zur ambulanten Nachsorge entlassen. Er war für 50 m mobil und im Ruhezustand beschwerdefrei.

Sechs Tage nach der Entlassung stellte sich der Patient mit zunehmender Dyspnoe, Giemen sowie leichtem Husten mit gelbem Sputum erneut vor. In der Untersuchung zeigte sich eine erhöhte Atemarbeit und diffuses Giemen über den gesamten Atemzyklus. Eine faseroptische Nasenendoskopie bis zur mittleren Trachea ergab keine paradoxen Stimmbandbewegungen und keine Obstruktion. In der Computertomographie (CT) zeigte sich eine weiträumige Verdickung der Bronchialwand von den großen bis zu den mittleren Atemwegen sowie eine leichte Milchglastrübung bilateral in den peripheren Oberlappen (Abb. 4). Eine Bronchoskopie ergab eine persistierende diffuse, fleckige Mucusauskleidung der Atemwege vor allem im linken Oberlappen, wo auch eine leichte Verengung zu sehen war, jedoch war das Gesamtbild wesentlich besser als bei der initialen Aufnahme; und die Lavage ergab wenige inflammatorische Zellen (vor allem Neutrophile) und keine Mikroben.

Der Patient wurde empirisch mit hochdosiertem Prednisolon, inhalativen Bronchodilatatoren und Antibiotika behandelt. Er litt erheblich unter Angst und wurde mit Sertralin und Olanzapin sowie Clonazepam nach Bedarf behandelt. Die Dyspnoe verschlechterte sich, und im Verlauf der nächsten 17 Tage traten zunehmende Kopfschmerzen und Schläfrigkeit auf. Der Befund lautete auf schwere, jedoch teilweise kompensierte Typ-II-Atemin-
46

Kompass Pneumol 2020;8:45-51 DOI: $10.1159 / 000505086$ 

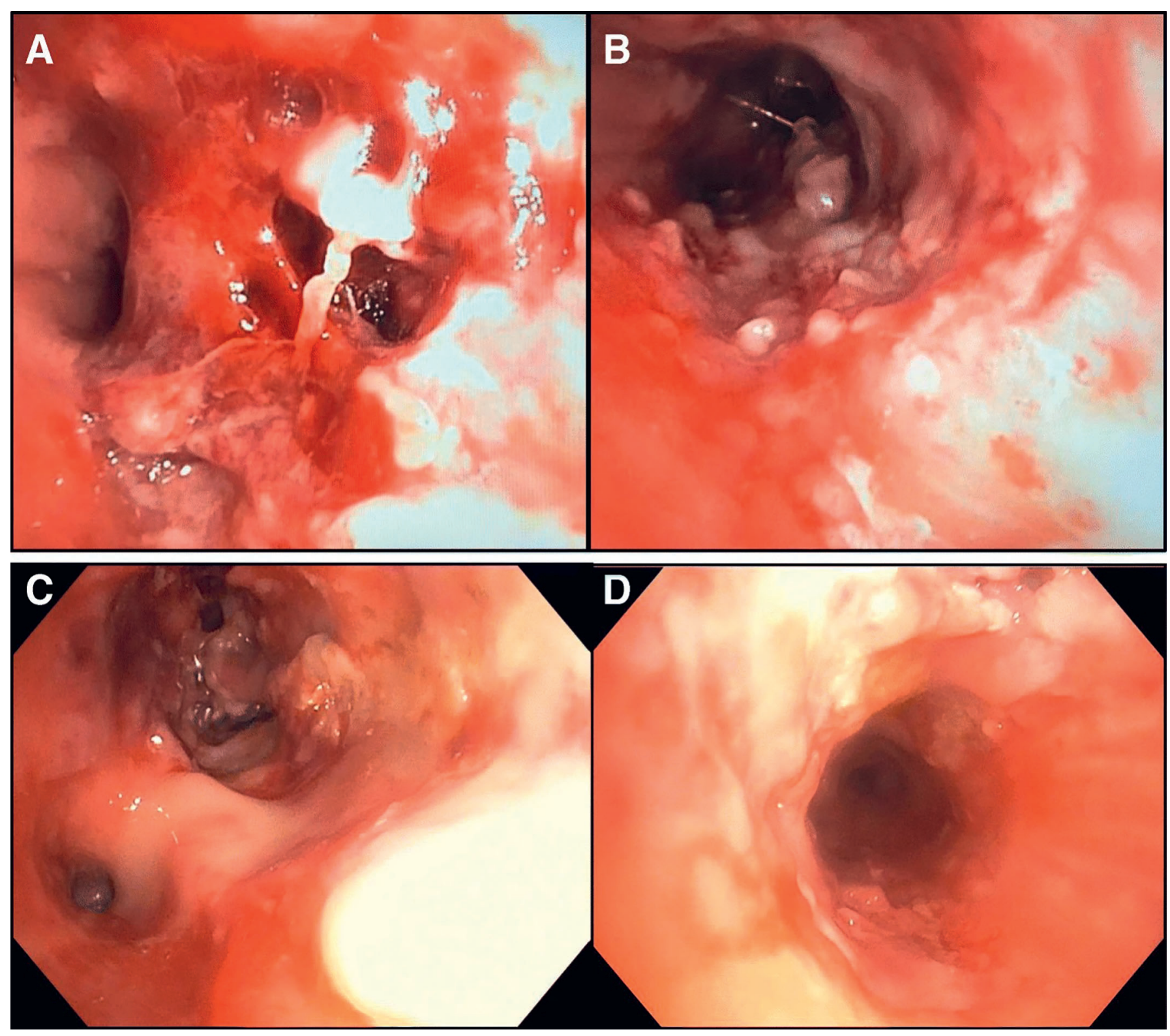

Abb. 3. 4 Aufnahmen der zentralen Atemwege aus der Bronchoskopie, die initial bei der Aufnahme durchgeführt wurde, zeigen eine schwere, diffuse Inflammation der Atemwege mit eitrigem Exsudat und Ulzera sowie Noduli. Bild (a) ist aus dem proximalen linken Oberlappenbronchus. Bild (b) ist aus der Trachea. Bild (c) zeigt den linken Unterlappenbronchus im unteren linken Teil und den linken Oberlappenbronchus im oberen mittleren Teil. Bild (d) ist aus dem rechten Hauptbronchus.

suffizienzbeinormaleralveolo-arterieller(Aa)Sauerstoffdifferenz (arterielle Blutgasuntersuchung bei $\mathrm{FiO}_{2}$ 0,27 ergab pH 7,33, $\mathrm{P}_{\mathrm{a}} \mathrm{O}_{2}$ $99 \mathrm{mmHg}, \mathrm{P}_{\mathrm{a}} \mathrm{CO}_{2} 74 \mathrm{mmHg}$, Bicarbonat $34 \mathrm{mmol} / \mathrm{l}$ ).

Die wiederholte CT-Bildgebung zeigte eine persistierende, aber abnehmende Bronchialwandverdickung, nun jedoch signifikante homogene Lufteinschlüsse (Abb. 5); es wurde angenommen, dass diese auf eine Bronchiolitis infolge der vermuteten anfänglichen Inhalationsverletzung zurückzuführen waren. Weitere erwogene Differenzialdiagnosen waren ein zentrales Defizit (neurologische Untersuchung und Magnetresonanztomographie des Gehirns waren unauffällig), Arzneimittelwirkungen (Absetzung der Benzodiazepine brachte keinen Nutzen) sowie neuromuskuläre Schwäche (Atemanstrengungen schien erheblich, mit kostalen Einzugs-/ paradoxen Bauchbewegungen, Anti-Acetylcholinrezeptor-Antikörper lagen nicht vor, und eine Elektromyographie mit Untersuchung der Erregungsleitung war unauffällig).

Der Zustand des Patienten verschlechterte sich trotz einer versuchten nicht invasiven Beatmung, zeigte Schläfrigkeit $\left(\mathrm{P}_{\mathrm{a}} \mathrm{CO}_{2}\right.$ $175 \mathrm{mmHg}$ ) und musste intubiert und maschinell beatmet werden. Die Beatmung gestaltete sich schwierig; es war ein inspiratorischer Spitzendruck von $69 \mathrm{~cm} \mathrm{H}_{2} \mathrm{O}$ erforderlich, um ein Atemzugvolumen von $3 \mathrm{ml} / \mathrm{kg}$ zu erreichen. Die Strömungsbehinderung in der Exspiration und Plateau-Atemwegsdrücke $<20 \mathrm{~cm}$ $\mathrm{H}_{2} \mathrm{O}$ deuteten auf einen vorwiegend obstruktiven Atmungsdefekt hin, und eine intermittierende Trennung vom System war erforderlich, um Lufteinschlüsse abzubauen. 
Eine Bronchoskopie während der maschinellen Beatmung ergab multiple konzentrische fibröse, netzartige Stenosen in den Lappen- und Segmentbronchien beider Lungenflügel. Teilweise waren die Bronchien dadurch vollständig verschlossen. Viele Stenosen konnten jedoch erfolgreich ballondilatiert werden, und dort, wo Membranen die Atemwege vollständig verschlossen, wurden diese mit der Wang-Nadel punktiert und dann ebenfalls aufgeweitet (Abb. 6). Stents stellten angesichts der großen Zahl der Stenosen und der Beteiligung nicht zentraler Atemwege keine Option dar. Unmittelbar nach dem Eingriff trat eine dramatische Verbesserungder Atemleistungein. DerinspiratorischerSpitzendruck ging auf $18 \mathrm{~cm} \mathrm{H}_{2} \mathrm{O}$ zurück, und der Patient wurde am nächsten Tag extubiert und benötigte nur noch minimale Sauerstoffgaben.

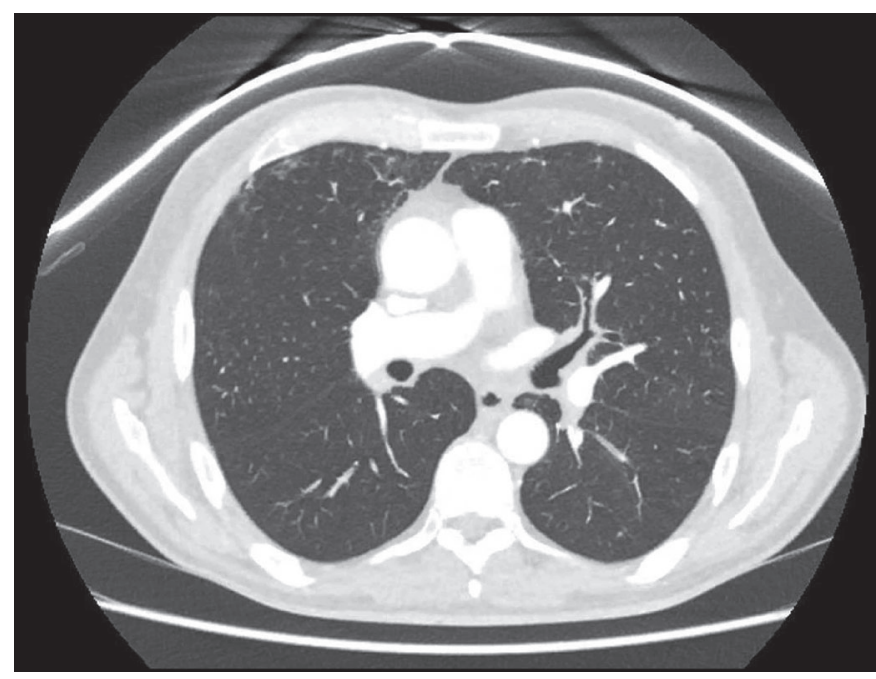

Abb. 4. CT-Schnittbild aus der bildgebenden Untersuchung des Thorax bei erneuter Vorstellung zeigt Bronchialwandverdickung, hier besonders deutlich im linken Oberlappenbronchus und proximalen Teil des Bronchus segmentalis anterior (LB3).
Endobronchiale Biopsien ergaben eine unspezifische Schleimhautulzeration und chronische Entzündung mit stromaler Fibrose. Es wurde vermutet, dass ein intensiv proliferierender/fibrosierender inflammatorischer Prozess infolge einer ungeordneten Schleimhautheilung nach der vermuteten initialen Inhalationsverletzung vorlag, daher wurde versuchsweise eine gepulste hochdosierte Methylprednisolon-Behandlung eingeleitet.

Der Zustand des Patienten verbesserte sich weiter, und der Patient wurde mit einem Plan zur ambulanten Rehabilitation entlassen, auf die nach 3 Wochen eine erneute Bronchoskopie folgen sollte. Die Spirometrie bei der Entlassung belegte eine mittelschwer bis schwer obstruktive Pathophysiologie und eine herabgesetzte Diffusionskapazität $\left(\mathrm{FEV}_{1}\right.$ 1,79 [49\% des Sollwerts], FVC 3,26 [70\% des Sollwerts], Hämoglobin-bereinigte Kohlenmonoxid-Diffusionskapazität 19,2 $\mathrm{ml} / \mathrm{min} / \mathrm{mm} \mathrm{Hg}$ [66\% des Sollwerts]). Eine Plethysmographie war wegen Klaustrophobie nicht möglich.

Nach 6 Tagen kehrte der Patient mit zunehmender Dyspnoe, Giemen und leichter Typ-I-Ateminsuffizienz zurück. Die Bronchoskopie zeigte ein aggressives Wiederauftreten der netzartigen Stenosierung mit Okklusion mehrerer Segmentbronchien, von denen einige mittels Punktion und Dilatation wieder durchgängig gemacht wurden. An mehreren Stellen hob sich die Schleimhaut vom darunter liegenden Bronchialknorpel ab, was weitere Interventionen unmöglich machte. Die Trachea schien weitgehend verschont zu sein und die proximalen Hauptbronchien nur minimal betroffen. Eine gründliche Anamnese auch unter Hinzuziehung kollateraler Quellen erfolgte im Hinblick auf eine mögliche inhalative Expositionen im heimischen Umfeld aufgrund der wiederholt nach der Entlassung rasch eintretenden Verschlechterung. Es konnte jedoch kein möglicher Verursacher identifiziert werden, mit Ausnahme von Malathion, das der Patient einige Tage vor seiner Erstvorstellung, danach jedoch nicht mehr, gegen Ameisen versprüht hatte. Da diese Exposition erst zu diesem spä-

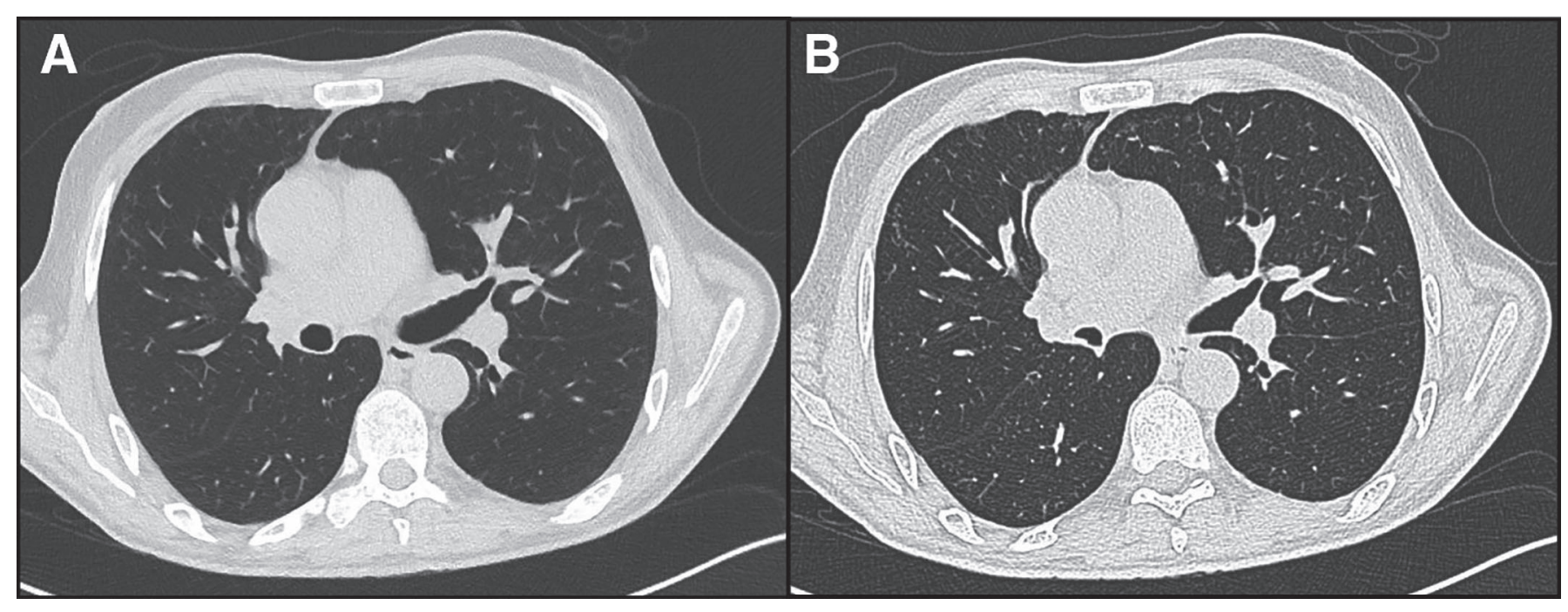

Abb. 5. 2 CT-Schnittbilder aus der bildgebenden Untersuchung des Thorax rund drei Wochen nach der erneuten Vorstellung, die linke Aufnahme (a) bei vollständiger Inspiration und die rechte Aufnahme (b) bei vollständiger Exspiration. Die Bilder zeigen eine leichte Besserung der in Abb. 4 dargestellten Bronchialwandverdickung im linken Oberlappenbronchus, vor allem jedoch wenig Veränderung bei der pulmonalen Transparenz in Exspiration wegen signifikanter homogener Lufteinschlüsse. 
teren Zeitpunkt bekannt wurde, konnten die Blutproben von der initialen Aufnahme nicht mehr auf die Cholinesterasekonzentration analysiert werden.

Angesichts der schweren und rasch wieder auftretenden multiplen Stenosierung sowie des zunehmend hohen Risikos endobronchialer Eingriffe wurde der Patient zügig für eine Lungentransplantation vorbereitet. Es gab Bedenken bezüglich der Transplantation wegen einer Atemwegserkrankung unbekannter Ursache - dass die zugrunde liegende Erkrankung systemisch sein könnte, dass die Anastomosen und nativen großen Atemwege betroffen sein könnten oder dass die Erkrankung auch im Transplantat wieder auftreten könnte. Außerdem war der Patient in schlechter Verfassung und nicht in der Lage, die Standard-Vorbereitung für eine Lungentransplantation zu durchlaufen. Die chirurgischen Optionen umfassten eine standardmäßige bilateral-sequenzielle Lungentransplantation sowie eine DominoHerz-Lungen-Transplantation mit Trachealanastomose. Einen Monat später wurde der erstgenannte Eingriff im Spezialzentrum der Quartärversorgung durchgeführt, und die pathologische Untersuchung des Explantats ergab eine sehr schwere nekrotisierende Bronchitis, die sich tief in die Wand hinein erstreckte und mit Granulation und Fibrosierung einherging, am schwersten in den zentralen Bronchien, mit einigen Bereichen der histiozytärer Inflammation insbesondere in der distalen Trachea; eine spezifische Diagnose konnte jedoch nicht gestellt werden. Zwölf Monate nach der Transplantation entwickelt der Patient sich gut, die Transplantatfunktion ist normal, und es gibt keine Anzeichen einer Rezidivierung oder systemischen Erkrankung.

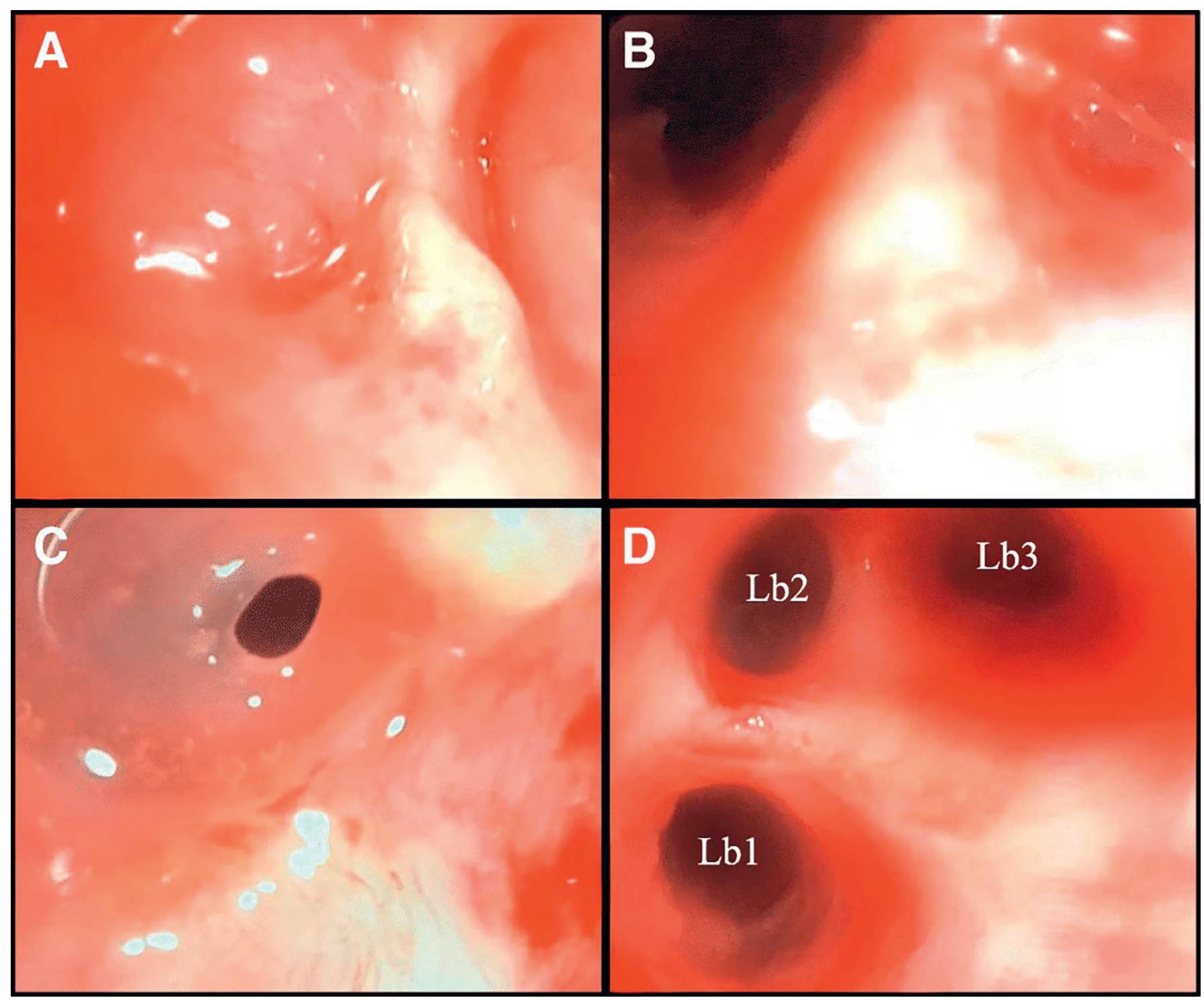

Abb. 6. Bronchoskopische Aufnahmen nach Verschlechterung des Patienten mit schwerer Typ-II-Ateminsuffizienz, die Intubation und maschinelle Beatmung erforderlich machte. Bild (a) zeigt die vollständige membranöse Okklusion von RB1b; Bild (b) zeigt dasselbe Bronchialsegment nach Eröffnung durch Punktion der Membranstenose mittels Wang-Nadel und anschließender Ballondilatation. Bild (c) zeigt eine nahezu vollständige Stenosierung im oberen Anteil des linken Oberlappenbronchus. Bild (d) zeigt den Blick jenseits der Stenose nach der Dilatation; hier zeigt sich eine Normvarianten-Konfiguration der linken Oberlappen-Segmentbronchien LB1/LB2/LB3. 


\section{Diskussion und Schlussfolgerungen}

Dieser Fall gibt Anlass zur Diskussion über die diagnostischen Lektionen, die aus der erneuten Vorstellung mit Typ-II-Ateminsuffizienz zu ziehen sind - als Beispiel für interventionelle pulmonologische Strategien, als sehr seltener Fall schwerer diffuser Bronchusstenosen nach vermuteter inhalativer Atemwegsverletzung und als extrem seltene Indikation für eine Lungentransplantation unter hohem Risiko.

Die Ursache für die schwere Typ-II-Ateminsuffizienz bei der Wiedervorstellung war zunächst unklar. $\mathrm{Zu}$ den betrachteten Differenzialdiagnosen zählten eine parenchymale Lungenerkrankung (jedoch war die Aa-Differenz normal und die Bildgebung sprach nicht dafür) und andere Möglichkeiten, die wie oben beschrieben ausgeschlossen wurden (Erkrankungen des zentralen Nervensystems, neuromuskuläre Erkrankungen, Medikamente). Aufgrund der homogenen Lufteinschlüsse in der Bildgebung wurde die Hypothese aufgestellt, dass eine initiale chemische Inhalationsverletzung eine Bronchiolitis obliterans hervorgerufen hatte.

Die Entdeckung ausgeprägter diffuser Bronchialstenosen bei der erneuten Bronchoskopie nur 17 Tage später lieferte eine bessere Erklärung für die Typ-II-Ateminsuffizienz und die Schwierigkeiten bei der Beatmung. Dies wurde durch eine dramatische Verbesserung nach bronchoskopischer Dilatation bestätigt. Wir folgern daraus, dass Kollateralventilation dafür sorgte, dass auch Bereiche distal von vollständig membranös verschlossenen Bronchialsegmenten weiterhin belüftet wurden. Eine feste und umfangreiche Atemwegsobstruktion bei vermindertem Luftstrom durch die Wege der Kollateralventilation deckt sich mit der Verzögerung sowohl bei der Inspiration mit hohem inspiratorischem Spitzendruck als auch bei der Exspiration und den beobachten Lufteinschlüssen und Hypoventilation.

Für das Auftreten einer oder einiger weniger Bronchialstenosen gibt es viele mögliche Ursachen. Bekannte Ätiologien sind unter anderem angeborene Läsionen, Infektionen (Mykobakterien, Diphtherie, Influenza, Papillomatose, Rhinosklerom, Pilze), Autoimmunerkrankungen (Granulomatose mit Polyangiitis, mikroskopische Polyangiitis, Sarkoidose, Morbus Crohn, Morbus Behçet, rezidivierende Polychondritis), Inhalationsverletzungen (thermische Verbrennungen, Senfgas, Salzsäure), gutartige und bösartige Tumoren, Krebstherapien (Strahlentherapie, endobronchiale Brachytherapie), Trauma (Langzeit-Intubation, vorausgehende Operation) und weitere Ursachen (Amyloidose, Tracheobronchopathia osteochondroplastica, vorausgehende Transplantation, idiopathisch) $[2,3,4,5,6]$.

Für multiple Bronchusstenosen sind weitaus weniger Ursachen identifiziert, und es gibt nur wenige Berichte in der Literatur. Mailloux und Kollegen berichten von einem Fall multipler netzartiger Bronchusstenosen, die nach einer schweren Erkrankung infolge einer Infektion mit Influenza-A (H1N1) auftraten; dieser Fall weist einige bemerkenswerte Gemeinsamkeiten mit unserem Fall auf, einschließlich der verzögerten Vorstellung nach der initialen Atemwegsschädigung [1]. Rubin et al. beschreiben einen
Fall multipler Stenosen der Trachea und der Haupt- und Lappenbronchien, die ebenfalls einen Monat nach einer initialen Atemwegsverletzung (in diesem Fall durch Inhalation von Salzsäure) auftraten [4]. Keating und Kollegen schildern einen Fall multipler netzartiger Bronchusstenosen, die 2 Jahre nach einer Lungentransplantation auftraten, ohne dass eine Ursache identifiziert wurde [6]. Ulloa-Clavijo et al. beschreiben einen Fall von multiplen netzartigen Bronchialstenosen bei einem jugendlichen Patienten mit Granulomatose mit Polyangiitis [3]. Mizushima et al. berichten von einem Fall multipler Stenosen der Lappenbronchien auf dem Boden einer Sarkoidose [7]. Genau wie in unserem Fall wird in vielen dieser Berichte beschrieben, dass die Bronchusstenosen erst mit erheblicher Verzögerung erkannt wurden, während eine Behandlung wegen vermutetem Asthma oder chronisch-obstruktiver Lungenerkrankung erfolgte; dies unterstreicht die diagnostischen Herausforderungen dieses seltenen Phänomens und belegt die Überlegenheit der Bronchoskopie gegenüber der CT-Bildgebung bei der Beurteilung von Atemwegsstenosen - insbesondere solchen mit netzartiger Konfiguration, die dünner als die CT-Schnittdicke sein können, aber dennoch eine schwere Atemwegsobstruktion verursachen.

Malathion ist ein Organophosphat-Pestizid, das als geringfügig akut-toxisch gilt. Seine Auswirkungen beim Menschen werden durch Hemmung der Acetylcholinesterase, Anreicherung von Acetylcholin und cholinerge Toxizität vermittelt. Malathion war das einzige inhalierte Toxin, das bei unserem Patienten identifiziert werden konnte, doch das von uns beobachtete klinische Bild ist nicht typisch für das, was in historischen Studien zur Toxizität von Malathion beim Menschen beschrieben wird [8]. Dieser Fall könnte zwar theoretisch eine neuartige Manifestation von Malathion-Toxizität darstellen, doch wahrscheinlicher scheint es, dass ein anderes, nicht identifiziertes Toxin inhaliert wurde oder dass die Krankheit auf einen nicht identifizierten Mikroorganismus oder einen entgleisenden Autoimmunprozess zurückzuführen war.

Wir gehen davon aus, dass es sich um einen seltenen Fall handelt, da eine Verletzung wie diese vor Beginn der ECMO-Ära letal gewesen wäre, und dass die Vielzahl der aufgetretenen Stenosen die Schwere der ursprünglichen Atemwegsverletzung widerspiegelt. Einerseits beleuchtet unser Fall, wie viel sich mit der ECMO erreichen lässt, andererseits ist der Fall ein Warnbeispiel dafür, welche Morbidität es nach sich ziehen kann, wenn man «das Unüberlebbare überlebt>.

\section{Verfügbarkeit von Daten und Materialien}

Alle relevanten Daten zu diesem Fall sind im vorliegenden Manuskript enthalten. Eine darüber hinausgehende Bereitstellung von Daten wird nicht in Betracht gezogen, da der Schutz der Privatsphäre des Patienten Vorrang hat.

\section{Finanzierung}

Die Erstellung der vorliegenden Arbeit wurde nicht mit externen Finanzmitteln unterstützt.
50

Kompass Pneumol 2020;8:45-51 DOI: $10.1159 / 000505086$ 


\section{Beiträge der Autoren}

TC und JL erhoben die klinischen Daten und verfassten den Manuskriptentwurf. AY führte eine gründliche Durchsicht des Manuskripts durch und steuerte atmungsphysiologische Aspekte bei. BR führte eine gründliche Durchsicht des Manuskripts durch und steuerte Aspekte zur Intensivmedizin und extrakorporalen Membranoxygenierung bei. PN führte eine gründliche Durchsicht des Manuskripts durch und steuerte Aspekte zur interventionellen Pneumologie bei. HW und CLHL führten jeweils eine gründliche Durchsicht des Manuskripts durch und steuerten lungentransplantologische Aspekte bei. Alle Autoren haben die finale Fassung des Manuskripts gelesen und freigegeben.

\section{Zustimmung zur Veröffentlichung}

Für die Veröffentlichung des vorliegenden Manuskripts und der dazugehörigen Bilder wurde die schriftliche Einwilligung des Patienten eingeholt.

\section{Interessenskonflikte}

Die Autoren erklären, dass keine Interessenskonflikte bestehen.

\section{Lizenzangabe}

Thomas Crowhurst, Joshua Lightfoot, Aeneas Yeo, Benjamin Reddi, Phan Nguyen, Helen Whitford, Chien-Li Holmes-Liew: Case report of severe bronchial web-like stenoses after «surviving the unsurvivable〉, BMC Pulm Med 2019;19(1):119 (DOI: 10.1186/s12890-019-0873-z). 을 ${ }^{\circ}$ The Author(s) 2019 (Übersetzung; Danksagung, Ethikerklärung und Publisher's Note gekürzt), lizensiert unter CC BY 4.0 (https://creativecommons.org/licenses/ by/4.0/deed.de)

\section{Literatur}

1 Mailloux B, et al.: Extensive web-like endobronchial membranous stenosis in a patient after H1N1 influenza a infection. CHEST 140(4):82A.

2 Prince JS, et al.: Nonneoplastic lesions of the tracheobronchial wall: radiologic findings with bronchoscopic correlation. Radiographics 2002;22:Spec No: p. S215-230.
Ulloa-Clavijo C, et al.: Web-like bronchial stenosis secondary to granulomatosis with polyangiitis. Reumatol Clin 2018;14(2):119-120.

4 Rubin AE, Wang KP, Liu MC: Tracheobronchial stenosis from acid aspiration presenting as asthma. Chest 2003;123(2):643-646.

5 Amat B, et al.: The electrosurgical knife in an optimized intermittent cutting mode for the endoscopic treatment of benign web-like tracheobronchial stenosis. Arch Bronconeumol 2012;48(1):14-21.
6 Keating DT, et al.: Proliferating bronchial webs after lung transplantation. Ann Thorac Surg 2011;92:1893-1896.

7 Mizushuma Y, et al.: Sarcoidosis accompanied by pleural effusion and multiple bronchial stenoses. Intern Med 1997;36(9):637-639.

8 Golz HH: Controlled human exposures to malathion aerosols. AMA Arch Ind Health 1959;19(5):516-523. 